\title{
NECESIDAD DE CIRCUNCISIÓN O DILATACIÓN DEL PREPUCIO. ESTUDIO DE 1.200 NIÑOS
}

\author{
Julio C. Morales Concepción', Pedro González Fernández², Aymée Morales Aránegui³. \\ Marlen Guerra Rodríguezl y Bárbara Mora Casacó'.
}

Hospitales Pediátricos Centro Habana'. William Soler². Juan Manuel Märquez³. Ciudad de La Habana. Cuba.

\begin{abstract}
Resumen.- OBJETIVOS: Demostrar el incremento de la retractilidad del prepucio con la edad. Señalar la poca utilidad de la circuncisión y la dilatación forzada del prepucio en la infancia.
\end{abstract}

MÉTODOS: El desarrollo del prepucio y su retractilidad, a medida que avanza la edad, fueron evaluados en 1200 niños de 0 a 16 años. El prepucio fue catalogado en tipos I a $\mathrm{V}$ según fue de menor a mayor su retractilidad en todos los casos que habían sido o no dilatados previamente.

RESULTADOS: La retractilidad del prepucio en los niños menores de un año en el tipo I (no retráctill) fue 63,4
$\%$, mientras en el tipo $\mathrm{V}$ (completamente retráctill) fue tan solo 3,7 \%. Lo contrario se observó en los adolescentes (1 1 a 16 años) en los que el tipo I fue 0,9 $\%$, mientras el tipo $V$ se observó en 80,9\%. También se encontró que 309 niños $(43,1 \%)$ de los 717 niños que habían sido dilatados forzadamente su prepucio, cuando eran mas pequeños, tenían prepucios tipos I al $I V$, es decir, habian adquirido nuevamente adherencias balano-prepuciales en el momento del examen para nuestra investigación. Se observó que 17 niños de los examinados $(0,4 \%)$ estaban necesitados de que se les realizara circuncisión. Ningún niño sufrió infección del tractus urinario superior.

CONCLUSIONES: Todos los niños nacen con el prepucio cubriéndole el glande, si no tienen una anomalía congénita del pene, manteniendo unas adherencias entre ambas estructuras, las que van desapareciendo con la edad, siendo total la separación en la pubertad, en la mayoría de los niños. Por ello consideramos la circuncisión o la dilatación forzada del prepucio innecesaria en la mayoría de los niños.

Palabras clave: Prepucio. Fimosis. Dilatación forzada. Circuncisión.

Summary.- OBJECTIVES: To demonstrate the increase of preputial retractability with age. To point out the small usefulness of circumcision and preputial forced dilation during childhood.

METHODS: The development of the prepuce and its retractability were evaluated in 1200 boys between 0 and 16 years. The prepuce was classified as type I to $V$ depending on its lower or higher retractability in all cases having been dilated previously or not. 
RESULTS: Prepuce retractability in boys under one year was type I (not retractile) in $63.4 \%$, whereas it was type $\checkmark$ (completely retractile) in only $3.7 \%$. The contrary was observed in adolescents 111 to 16 years), in which type I was $0.9 \%$ and type $V$ was observed in $80.9 \%$. It was also observed that 309 boys (43.1\%) among the 717 with previous prepuce forced dilation, had types I to IV prepuces, so, they had acquired new balanopreputial adherences by the time of examination for our study. Seventeen boys (0.4\%) required circumcision. No children suffered upper urinary tract infections.

CONCLUSIONS: All boys are born with the prepuce covering the glans penis, keeping adherences between both structures, which disappear with age, being the detachment complete at the time of puberty in most boys. So, we consider circumcision or forced dilation of the prepuce unnecessary in most boys.

Keywords: Prepuce. Phimosis. Forced dilation. Circumcision.

\section{INTRODUCCIÓN}

El prepucio es la parte de la piel del pene que recubre el glande, si no existe una anomalía congénita en el mismo. Hace muchos años se consideraba que el prepucio favorecía las infecciones urinarias en la infancia $(1,2)$. También se creía que el prepucio predisponía a las enfermedades venéreas y al cáncer del pene $(3,4)$, por la irritación a las mucosas que produce la acumulación de esmegma entre el prepucio y el glande, al igual que producir dificultades en el normal desenvolvimiento de las relaciones sexuales.

Todos estos argumentos han sido la base para indicar la circuncisión o la dilatación forzada del prepucio como medida profiláctica en los niños pequeños. No obstante, estos criterios han tenido opiniones contrapuestas, basadas en investigaciones muy concienzudas (5-12). Siempre mantuvimos el criterio que la circuncisión post-natal indiscriminada en todos los varones (que estaba muy difundida en nuestro país, sobre todo por los gineco-obstetras), era una medida muy exagerada. Por esa época llevamos a cabo una investigación que demostró que en el $80 \%$ de los niños se podía realizar la dilatación forzada del prepucio, lo que considerábamos apropiado en esos momentos, sin tener que acudir a la circuncisión, facilitando con ello el aseo diario del glande y el prepucio (13).
Por todo lo expuesto, y motivado por una investigación que revisamos (11), nos dimos a la tarea de describir el desarrollo del prepucio y su retractilidad, a medida que avanzaba la edad, en 1200 niños de tres hospitales pediátricos de Ciudad de la Habana, Cuba, y de esta forma señalar realmente la necesidad de la circuncisión o la dilatación forzada del prepucio en nuestro grupo de pacientes.

\section{MATERIAL Y MÉTODO}

Los autores llevaron a cabo examen del pene de todos los niños varones que concurrían a la consulta externa de la especialidad o estaban ingresados en salas de pediatría de los hospitales pediátricos universitarios de Centro Habana, William Soler y Juan Manuel Märquez en Ciudad de La Habana, Cuba. Se tuvo en cuenta sólo a los niños que no habían sido circuncidados o que su prepucio no hubiese sido ampliado por cualquier medio señalando tan solo si su prepucio había sido dilatado o no cuando eran mas pequeños. Con los niños en posición supina, lo mas relajado posible, el prepucio fue suavemente retraído, sin traumatismos, señalando el grado de retractilidad y la existencia o no de adherencias balano prepuciales.

El estado prepucial se consideró en 5 tipos basados en su retractilidad y de acuerdo con la clasificación de Kayaba y cols. (1 1):

Tipo I: leve retractilidad sin que se vea el glande;

Tipo II: exposición del meato uretral con retracción ligeramente mayor del prepucio;

Tipo III (intermedio): con exposición del glande hasta la parte media de él, pero aun se observaban algunas adherencias en el resto del mismo;

Tipo IV: exposición del glande hasta la corona, pero aun se observaban adherencias en la misma y

Tipo V: cuando existía exposición fácil de todo el glande y el surco balano prepucial sin adherencias. (Figura 1).

Fueron señalados en todos los casos los que habían sido dilatados su prepucio como se acostumbra en nuestro país cuando se les aplica el esquema de vacunación.

\section{RESULTADOS}

El grado de retractilidad prepucial se incrementó con la edad (Tabla I). Los niños con edad $<1$ año tuvieron prepucio tipo 1 (no retráctil) en $63,4 \%$ y tipo $\mathrm{V}$ (completamente retráctil) en 3,8\% respectivamente. En los niños con edades puberales $\left(\begin{array}{lll}1 & 1 & \text { a } \\ 16\end{array}\right.$ 


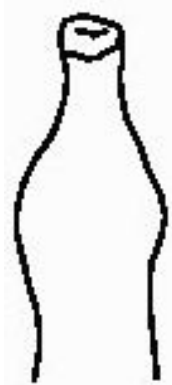

Tipo I

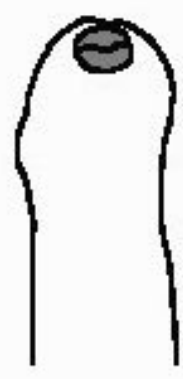

Tip o ॥

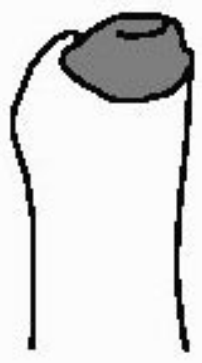

Tipo III

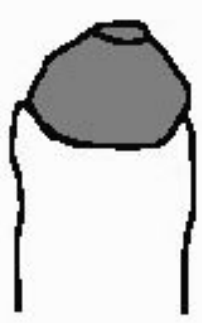

Tipo IV

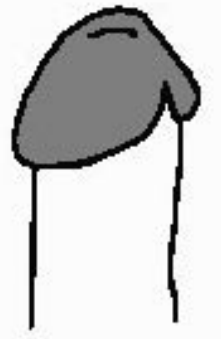

Tipo V
FIGURA 1. Tipos de prepucio, según la clasificación de kayaba y otros.

años) fue tipo I (no retráctil) $0,9 \%$ mientras el tipo $\mathrm{V}$ (completamente retráctil) fue $80,9 \%$. También se encontró que 309 niños $(43,1 \%)$ de los 717 que habían sido dilatados, tenían prepucios tipos I al IV con evidentes adherencias balano prepuciales nuevamente.

Ningún niño sufrió parafimosis ni los padres manifestaron que hubieran sufrido infecciones del tractus urinario superior.

De los 1.200 niños se encontró que 17 (0,4 \%) necesitaban circuncisión: 6 por poseer balanitis xerótica; 4 por padecer balanopostitis a repetición al sufrir de diabetes, 6 por tener anillo prepucial estenótico que impedía la micción normal, provocando saco prepucial por acúmulo de orina y 1 al poseer anillo estenótico subprepucial que no permitiría la futura retractilidad del prepucio. Todo ello constituyó solamente el 1,4\% de los 1200 niños examinados.

\section{DISCUSIÓN}

En la mayoría de los recién nacidos se observa una imposibilidad de retraer el prepucio hasta descubrir completamente el glande. Esta imposibilidad, que puede denominarse fimosis fisiológica, se mantiene durante los primeros 3-4 años de la vida,
TABLA I. RETRACTILIDAD DEL PREPUCIO*. PACCIENTES ESTUDIADOS: 1200. EDAD: O A 16 AÑOS

\begin{tabular}{|l|c|c|}
\hline 309 NIÑOS CON DILATACIÓN PREVIA: TIPO I-IV \\
\hline NIÑOS < 1 AÑO & $63.4 \%$ & TIPO I \\
\hline NIÑOS < 1 AÑO & $3.7 \%$ & TIPO V \\
\hline NIÑOS 11-16 AÑOS & $0.9 \%$ & TIPO I \\
\hline NIÑOS 11-16 AÑOS & $80.9 \%$ & TIPO V \\
\hline
\end{tabular}

*Tipo de prepucio según kayaba y cols. J. Urol. 156: $1813,1996$.

a partir de lo cual, diversos factores propician la separación entre ambas estructuras. Los factores que se han señalado son: el crecimiento anatómico del pene, la acumulación de bridas epiteliales entre el prepucio y el glande, las erecciones intermitentes del pene y la masturbación durante la pubertad. (14). Desde hace algunos años un autor europeo (5) describió que cerca del $90 \%$ de los prepucios se tornan retráctiles a los tres años de edad, sugiriendo que el conocer la historia natural del prepucio elimina el innecesario trauma manual o quirúrgico del pene que constituyen la dilatación forzada o la circuncisión. Este autor señaló que la lenta separación del prepucio y el glande coincide con la edad de la incontinencia urinaria y fecal del niño, realizando el prepucio una acción protectora, al haber notado que la ulceración del meato uretral sólo se observa en niños que han sido circuncidados. En ese mismo trabajo leímos la analogía propuesta por un autor anónimo, señalando que el prepucio protege al glande como el párpado lo hace al ojo.

Durante el crecimiento embrionario el prepucio aparece como un anillo de epidermis firme a las seis semanas de gestación, creciendo hasta el extremo del glande hasta las 16 semanas de embarazo. La individualización del prepucio y el glande comienza a las 24 semanas de gestación, avanzando hasta el nacimiento, pero con grandes variaciones según los individuos. Así algunos autores plantean que esta individualización será suficiente a los 10 días de nacido, lo que permite retraer el prepucio sin traumatizar el epitelio (14). Sin embargo Kayaba y 
cols. (11) encontraron el mismo grado de dificultad para retraer el prepucio en todos los niños japoneses menores de un año y que poseían los grados I a IV de su clasificación. Ello también se corrobora en nuestra investigación, la que contiene el mayor número de niños de las publicadas hasta hoy, encontrando que el $63,4 \%$ de los niños menores de un año tenían el prepucio tipo I (no retráctil). Oster (10) investigó el desarrollo prepucial en niños de 6 a 17 años en $\mathrm{Di}$ namarca, incluyendo 173 que fueron seguidos durante 7 años, hallando que la incidencia de adherencia prepucial decreció de $70 \%$ a los $6-7$ años a $5 \%$ a los 16-17 años. Estos hallazgos, unidos a los de nuestra investigación, señalan, inobjetablemente, que la separación incompleta del prepucio y el glande es normal y natural en recién nacidos y lactantes, progresando hasta hacerse total, en la mayoría de los niños, cuando han llegado a la pubertad. Estas adherencias, como ya señalamos, algunos autores $(1,2)$ la han considerado responsable de la colonización por microorganismos patógenos, lo que puede conducir a balanopostitis e infección urinaria alta. No obstante señalan que la pobre higiene genital en pre-escolares es más responsable de la balanitis que las adherencias balanoprepuciales. A pesar de ello consideran haber visto menos incidencia de infección urinaria en niños circuncidados que en los incircuncisos.

Esto pudiera ser, en algunos casos, una razón beneficiosa, pero nunca para justificar la circuncisión neonatal indiscriminada, pues ninguna de las investigaciones que hacen esos señalamientos, ha demostrado que los microorganismos causantes de la infección urinaria alta, pudieran ser los mismos encontrados en la infección prepucial de niños normales. Además es difícil de explicar la patogenia de estas infecciones en el varón, con los medios anatómicos defensivos que posee, superiores a la hembra, para impedir la progresión de las bacterias desde el prepucio hasta el riñón. Se considera que una adecuada higiene de las manos y genitales de los niños de esas edades puede prevenir estas infecciones.

Oster y Kayaba $(10,11)$ consideran que se necesitan estudios aleatorios para distinguir entre fimosis fisiológicas y patológicas. El considerar que la circuncisión rutinaria previene la incidencia de cáncer del pene (4) ha sido refutada por Oster (10) quien la encontró muy baja en países escandinavos, donde no se acostumbra a hacer la circuncisión neonatal indiscriminada, pero la higiene del glande y prepucio es siempre excelente en el adulto, impidiendo el acúmulo de esmegma, que es realmente lo que motiva la irritación de las mucosas de esas estructuras, facilitando la degeneración cancerosa (15). Ello fue igualmente refutado por Tranterberg y Gildin (16) en una magnífica comunicación que nos obsequiaron a raíz de nuestro trabajo, donde demostrábamos que la circuncisión neonatal carecía de sentido (13).

También Laumaun y cols. (17) consideran incorrectas estas aseveraciones. Muchas comunicaciones han aparecido en los últimos años con respecto a los trastornos psicológicos que la circuncisión neonatal acarrea. El dolor que la misma produce, al hacerse casi siempre sin anestesia alguna, y los cambios observados en la interacción materno-infantil, después de la circuncisión, aumenta la posibilidad de daños psicológicos en la madre y el niño (18). Taddio y cols. (19) señalan que los niños que han sido circuncidados al nacimiento, lloran mas intensamente y más tiempo, que los no operados, en el momento de imponerles las vacunas.

Maguire (20) informa que los trastornos psicológicos provocados por la circuncisión neonatal pueden detener el desarrollo neuropsicológico del niño, pudiendo propiciar alteraciones de la función cerebral y su desarrollo, conllevando a alteraciones de la conducta sexual cuando son adultos. Freud, el famoso psicoanalista, fustiga tremendamente ese tipo de circuncisión, señalando que la misma es un substituto de la castración (21). Además de los riesgos psicológicos que se han descrito, también se argumentan diversos riesgos anatómicos derivados de las circuncisiones.

Se ha señalado que la resección del prepucio amputa casi todos los receptores de la sensibilidad que el mismo posee (22), endurece la capa epitelial del glande expuesto, reduciendo la movilidad peneana durante el coito, provocando diferentes conductas de preferencia sexual (23). También se ha señalado que puede ocurrir ligadura de la arteria del frenillo prepucial, al elongarse este, lo que se acostumbra hacer frecuentemente durante la circuncisión, produciendo isquemia y posterior estenosis del meato en 5 a $10 \%$ de los casos (23). Igualmente se menciona que puede ocurrir la amputación de parte o todo el glande durante el acto quirúrgico (24).

Otras funciones le han sido señaladas al prepucio, además de la señalada protección al glande (5). Se considera que contribuye al placer sexual, a la lubricación vaginal y a la dinámica del movimiento por la fricción, que la piel prepucial enrollada, produce en la vagina, además de la estimulación durante los juegos sexuales y la masturbación (17). Se señala que es una estructura andrógeno dependiente con un complejo sistema de enzimas intradérmicas $(25,26)$. Se ha planteado, como ya se ha dicho, que la circuncisión sin una causa médica, que lo justifique, puede conllevar a síntomas psíquicos de mutilación genital (15-17). 
Múltiples trabajos se han publicado, proponiendo la aplicación de anestésicos locales para minimizar los trastornos psicológicos que el dolor produce durante la circuncisión sin anestesia, al igual que el aumento de la respuesta de esteroides (27-31). Después que la Academia Americana de Pediatría (32) estableció ciertos requisitos para que la circuncisión se lleve a cabo, múltiples han sido los trabajos que han aparecido en la literatura oponiéndose a la circuncisión de rutina, sin una causa médica que lo avale, exigiendo actualmente cualquier cirujano el consentimiento escrito de los padres del niño que será sometido a la operación. Del mismo modo son frecuentes las asociaciones que se han creado defendiendo el derecho de los niños a no ser mutilados con la circuncisión (33-39).

Coincidimos con Kayaba (11) y Wallerstein (39) que en ausencia de trastornos médicos reales - emergentes, el prepucio debe permanecer intacto, concepto que teníamos hace muchos años (13), y que se ha visto reforzado con la presente investigación y la opinión de múltiples investigadores que afirman que en la mayoría de los niños la separación del prepucio y el glande continúa como un proceso natural hasta la adolescencia en que es total en casi todos los sujetos $(5-8,10-14,32,39)$. Mientras la cirugía debe considerarse imprescindible en los adultos con fimosis patológica, en los niños solo debe realizarse en presencia de fimosis real, asociada con disuria, balanopostitis recidivante, y en algunos casos en que se haya producido una parafimosis, no siendo lógico circuncidar un niño por la única razón de tener poca retractilidad en el prepucio.

\section{CONCLUSIONES}

Consideramos haber demostrado, con nuestra investigación, que todos los niños nacen con el glande cubierto por el prepucio lo que paulatinamente desaparece con el crecimiento, siendo total en la pubertad en la mayoría de los niños. Por ello no creemos necesario la dilatación prepucial y, menos aun, practicar la circuncisión por observar que el prepucio no descubre totalmente el glande.

\section{BIBLIOGRAFÍA y LECTURAS RECOMENDADAS (*lectura de interés $y^{* *}$ lectura fundamental)}

1. HOGUERAS, M.; LARDELLI, P.; MARTÍNEZ, J.L. y cols.: "Epidemiología de las urgencias urológicas en nuestro hospital”. Actas Urológicas Españolas, 16: 769, 1992.

2. WISWELL, T.E.; ROSCELLI, J.D.: “Corroborative evidence for the decreased incidence of the urinary tract infections in circumcised male infants". Pediatrics, 78: 96, 1986.

3. HUTCHINSON, J.: "On the influence of circumcision in preventing syphilis". Medicine Tems Gazette, 32: 542, 1855.

4. WOLBARST, A.L.: "Circumcision in infancy: A prophylactic and sanitary measure". Am. Med., 32: 23, 1926.

5. GAIDNER, D.: "The fate of the foreskin". Br. J. Med., 2: 1433, 1949.

6. DUMSMIR, W.D.; GORDON, E.M.: "The history of the circumcision". BJU International, 83: $1,1999$.

7. DEWAN, D.A.; TIEU, H.C.; CHIENG, B.S.: "Phimosis: Is circumcision necessary?". J. Paediatr. Child Health, 32: 285, 1996.

8. VAN HOWE, R.B.: "Variability in penile appearance and penile findings: A prospective study". Br. J. Urol., 80: 776, 1999.

9. FERGUSON, D.M.; LAUTON, J.M.; SHANNON, F.T.: "Neonatal circumcision and penile problems: An 8 year longitudinal study". Pediatrics, 81: 537, 1988.

10. OSTER, J.: "Further fate of the foreskin. Incidence of prepucial adhesions, phimosis, and smegma among Danish school boys". Arch. Dis. Child., 43: 200, 1968.

11. KAYABA, H.; TAMURA, H.; KITAJURA, S. y cols.: "Analysis of shape and retractibílity of the prepuce in 603 japanese boys". J. Urol., 156: 1813, 1996.

12. SHANKAR, K.R.; RICKWOOD, A.M.K.: "The incidence of phimosis in boys". B. J. Urol., 84: 101, 1999.

13. MORALES, J.C.: "Usos y abusos de la circumcision”. Rev. Cub. Ped., 2: 31, 1963.

14. ELDER, J.S.: "Congenital anomalies of the genitalis". Campbell's Urology, 6th edition. Edited by P. C. Walsh et al. Philadelphia; W. B. Saunders C. Vol. 2, chap. 51, 1920-1938, 1992.

15. CASON, D.; CARTER, B.; BATH, A.J.: "Can circumcision prevent recurrent urinary tract infection in hospitalized infants?" Clin. Pediatr., 699, 2000.

16. TRACTEMBERG, M.; GILDIN, L.R.: "El falso valor profiláctico de la circuncisión en la prevención del cáncer cervical y del pene. Su significado 
de mutilación genital y psíquica en niños y adultos". Comunicación personal, 1961.

17. LAUMAN, E.O.; MASI, C.M.; ZUCKERMAN, E.W.: "Circumcision in the United States: Prevalence, prophylactic effects and sexual practice". JAMA, 277: 1052, 1997.

18. GOLDMAN, R.: "The psychological impact of circumcision". BJU International, 831: 93, 1999.

19. TADDIO, A.; KATZ, J.; HERSICH, A. y cols.: "Effect of neonatal circumcision on pain response during subsequent routine vaccination". Lancet, 349: 599, 1997.

20. MAGUIRE, P.: "Coping with loss, surgery and loss of body parts". Br. Med. J., 316: 1086, 1998.

21. FREUD, S.: "Introductory lectures of psychosomatics". Strachey J. Ed. And trans, 1920; reprint New York Norton., 165, 1966.

22. MAIZELS, M.: "Normal development of the urinary tract". Campbbell"s Urology, 6th edition. Edited by P. Walsh P.C., Retic AB, Stamey TA Jr., Waugman Ed. Philadelphia: W. B. Saunders Co. Vol.2, chapt 32: 301-343, 1992.

23. PERSAD, R.; SHARMA, S.; McTAVISH, J. y cols.: "Clinical presentation and pathophysiology of meatal stenosis following circumcision". Br. J. Urol., 75: 91, 1995.

24. STRIMEING, B.S.: "Partial amputation of the glans penis during Mogen clamp circumcision". Pediatrics, 97: 906, 1996.

25. O'HARA, K.; O'HARA, O.: “The effect of male circumcision on the enjoyment of the female partner". BJU International, 83: 79, 1999.

26. COLD, C.J.; TAYLOR, J.R.: "The prepuce". BJU International, 1: 34, 2999.

27. TADDIO, A.; POLLACK, N.; GILBERT, R. y cols.: "Combined analgesia and local anesthesia to minimize pain during circumcision". Arch. Pediatr. Adolesc. Med., 154: 620, 2000.

28. HOWARD, C.R.; HOWARD, F.M.; GARFUNKEL, L.C. y cols.: "Neonatal circumcision and pain relief: Current training practices". Pediatrics, 101: 423, 1998.

29. KUYA, C.; WEITMAN, M.W. Jr.: "Neonatal circumcision and penile dorsal nerve block: A painless procedure". J. Pediatr., 92: 998, 1998.

30. HOWARD, C.R.; HOWARD, F.M.; WEITZMAN, M.L.: "Äcetaminophen analgesia in neonatal circumcision: The effect on pain". Pediatrics, 93: 641, 1994.

31. STANG, H.J.; GUNNAR, M.R.; SUELLMAN, L. y cols.: "Local anesthesia for neonatal circumcision: Effects on distress and cortisol response". JAMA, 2259: 1507, 1988.

32. AMERICAN ACADEMY OF PEDIATRICS.: "Task Force on Circumcision. Circumcision Policy Statement". Pediatrics, 103: 686, 1999.

33. NOBLE, E.; SORGER, L.: "The joy of being a boy". Library of Congress, catalog card number 94-66512.

*34. NATIONAL ORGANIZATION OF CIRCUMCISION INFORMATION RESOURCES CENTER. www. Nocirc. Org/

*35. DOCTORS OPPOSING CIRCUMCISION. faculty. washington.edu. gcd/DOC/

*36. ATTORNEYS FOR THE RIGHTS OF THE CHILD. www.arclau.org./

*37. STUDENTS FOR GENITAL INTEGRITY. www. studentsforgenitalintegrity.org/

38. MOTHERS AGAINST CIRCUMCISION. Www. othersagainstcircuncision.org/

39. WALLERSTEIN, E.: "Circumcision. The uniquely America Medical Enigma". Urol. Clin. N. Amer., 12: 125, 1985. 